\title{
Assessment of Qinling Forest Park's Ecological Security Based on PSR Model - A case study of forest parks in Baoji section of the Qinling Mountains
}

\author{
Zuo Ying ${ }^{1}$, Cui Yan ${ }^{2}$ \\ ${ }^{1}$ College of Urban and Environmental Sciences, Northwest University, Xi'an, China, 710127 \\ ${ }^{2}$ School of Architecture and Civil Engineering, Xi' an University of Science and Technology, Xi'an, China, 710054
}

\begin{abstract}
Green growth" is a generalized trend of growth poles in the new era. Ecological security is essential to the forest park tourism. The assessment research on ecological security is significant for the development of forest park tourism. From the perspective of ecological security, this thesis selects seven forest parks in Baoji section of the Qinling Mountains as the research object, and builds the assessment indicator system of forest park ecological security according to experts' comments. In this thesis, the PSR model is adopted to search indicators that slightly affect the security indexes of forest park; as a result ,outstanding problems are found in the forest park according to current conditions, such as weak protection of forest landscaping and environment, large density of forest buildings, low comprehensive management capacity and poor guidance of forest ecological tour. The thesis has also probed the ecological security protection and development path of forest park in the Qinling region from the perspective of tourism carrying capacity control, landscaping design and building control, park ecological management system, and tourist civilized tourism according to the common security risks in forests, hoping to provide reference for the ecological security protection of other forest parks in Qinling region.
\end{abstract}

\section{1 introduction}

Qinling (Qingling Mountains) is hailed as the winding mountain range of Chinese civilization and the center of geographical boundary between the country's north and south. Due to rich forest resources, unique landscaping and beautiful ecological environment, Qinling becomes a forest ecological tourism destination in Shaanxi Province. In February 2015, when General Secretary Xi Jinping made investigation in Shaanxi, he pointed out that this natural ecological landscape might not be damaged by anybody. This statement defines the direction of ecological and environmental protection in all cities and counties in Qinling region, provides solid policy guarantee for ecological resource protection and ecological tourism development in all forest parks in Qinling, and also drives the economic and social development in Qinling region due to popular ecological leisure and holiday tourism on the basis of forest $\mathrm{s}^{[1]}$. However, there are still many contradictions between the ecological tourism resource development in Qinling and ecological and environmental carrying capacity of all forest parks. The reduction and deterioration of biological diversity, decrease of service function of forest ecological system, unreasonable planning of forest parks and irrational development of parks have caused severe ecological and environmental problems such as excessive construction of infrastructure and tourism service facilities, ecological and environmental fragmentation and pressure as well as environmental pollution, etc. Moreover, Qinling Mountains are featured by complex geographical conditions vulnerable to disasters. Many phenomena have alerted us to the ecological and environmental protection of Qinling ${ }^{[3-5]}$. This thesis selects seven forest parks in Baoji section of the Qinling Mountains to study the environmental protection measures of ecological tourism in forest parks on the basis of ecological security evaluation, and provide reference for ecological and environmental guarantee in all forest parks and natural conservation regions in Qinling.

\section{Relevant research on ecological security assessment of forest parks}

Forest is the second ecological system in the world, which is featured by complex system elements and multiple ecological system functions. The forest ecological security refers to a state in which the ecological and natural environment during the survival and development of forest parks is free from or little imposed to damage or threat. The Organization for Economic Co-operation and Development adopts PSR model to evaluate the actual cases at first. There are many studies based on forest ecological security, which mainly focus on forest security index, forest ecological system monitoring, forest landscaping health, etc., but there are few studies on ecological security assessment on forest parks. For

\footnotetext{
* Corresponding author: 87086559@qq.com
} 
example, Tang adopts PSR concept model to evaluate the ecological security of Zijin Mountain National Forest Park on the basis of ecological security theory ${ }^{[6]}$; Mi et al. evaluate the ecological security in Jiu Peak National Forest Park in Beijing, and propose the protection and management countermeasures of sustainable development ${ }^{[7]}$; Wang analyzes the main factors that affect ecological security of forest parks, takes national and province-level forest parks in Wenzhou for example, and proposes the evaluation criteria on ecological security of forest parks $^{[8]}$

In above articles, the concept of forest park ecological security assessment is misused as the concept of forest ecological security. More attention shall be paid to the influence of tourists and park construction on forest ecological security. This study selects seven forest parks as specimens to conduct research on ecological security of Qinling forest park and in-depth exploration into the ecological security of forest park with PSR model from many perspectives.

\section{Brief information of research area}

The conservation area in Baoji section of the Qinling Mountains covers $8444.57 \mathrm{~km}^{2}$, accounting for $46.6 \%$ of the whole city. This area consists of three districts and four counties, including Weibin District, Chen Cang District,
Gaoxin District, Qishan County, Mei County, Feng County and Taibai County. It is also composed of more than 30 towns. The population in the area is more than 500,000 people. Baoji is located on the north of Qinling and in the middle of the Wei River. Its geological structure is complex, most of which is mountains and hills. The main peak is called Taibai Mountain which is 3,771.2 meters high. The climate is sub-humid warm temperate. It is rich in sunshine, heat and water resources. The annual sunshine duration is $1860-2250$ hours. The annual average precipitation is $590-900 \mathrm{~mm}$. It is an area with the most precipitation in Guanzhong region. Qinling is the boundary of rivers which belong to the river system of Yellow River and Yangtze River. There are many wildlife species, including 47 species of animals under state protection such as panda and Nipponia Nippon and more than 2300 types of wild higher plants including 17 types of plants under state protection such as ginkgo and Taxus chinensis, etc.

The Baoji section of the Qinling National Forest Park mainly includes Taibai Mountain National Forest Park, Tongtian River National Forest Park, Taibaiqingfeng Forest Park. The province-level forest parks mainly include Honghegu Forest Park and Zibai Mountain Forest Park. The municipal forest park is Xueshandong Forest Park. The geographical location of forest parks in the research area can be seen in Figure 1, and the basic information of forest parks is given in Table 1.

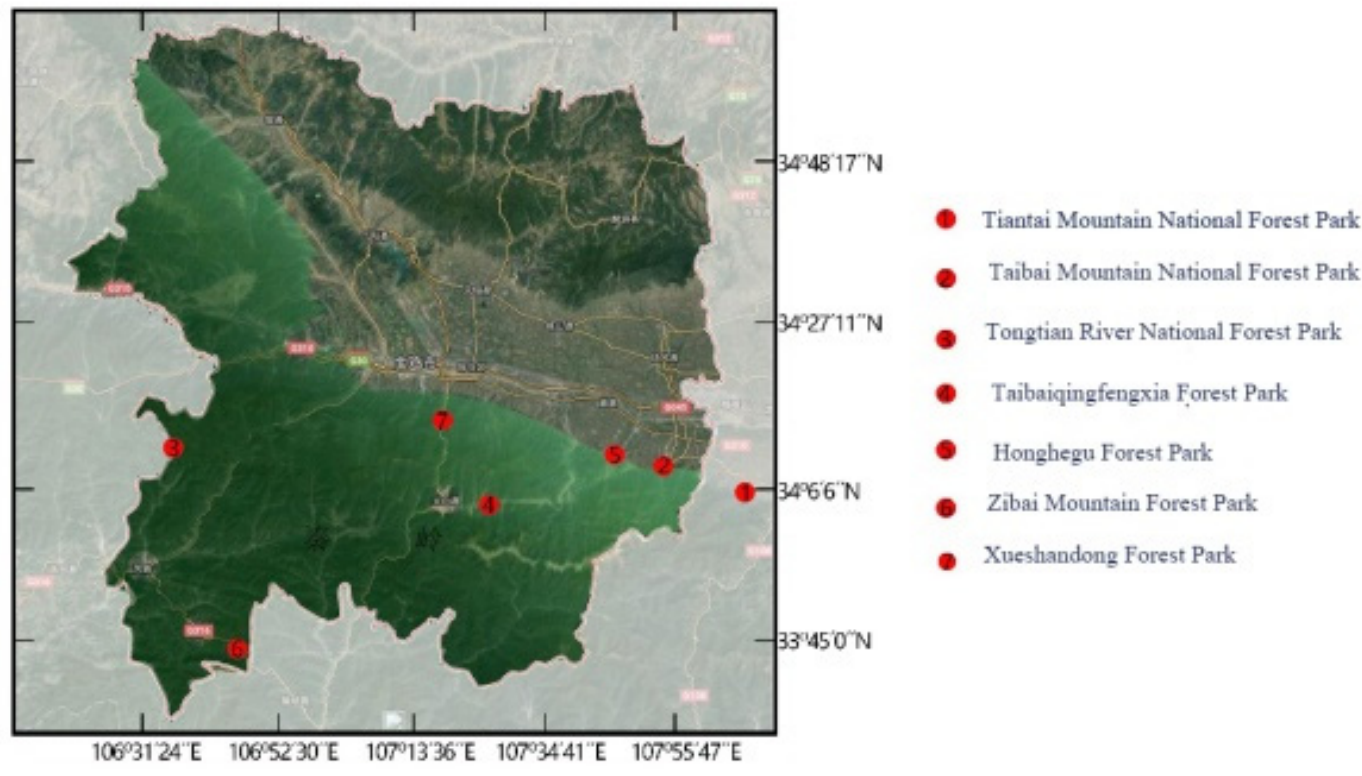

Figure 1. Geographical location map of forest parks in the study area 
Table 1. Overview of forest parks in the study area

\begin{tabular}{|c|c|c|c|c|c|c|}
\hline $\mathrm{SN}$ & Name & Grade & 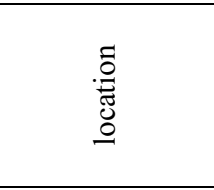 & 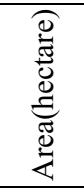 & 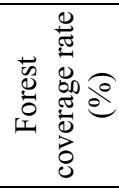 & $\begin{array}{l}\bar{\Xi} \\
\stackrel{d}{\lrcorner}\end{array}$ \\
\hline 1 & $\begin{array}{c}\text { Tiantai Mountain National Forest } \\
\text { Park }\end{array}$ & National & Weibin District & 3674 & 80 & $3 \mathrm{~A}$ \\
\hline 2 & $\begin{array}{c}\text { Taibai Mountain National Forest } \\
\text { Park }\end{array}$ & National & Mei County & 2949 & 94.3 & $5 \mathrm{~A}$ \\
\hline 3 & $\begin{array}{c}\text { Tongtian River National Forest } \\
\text { Park }\end{array}$ & National & Feng County & 5235 & 98.6 & $4 \mathrm{~A}$ \\
\hline 4 & Taibaiqingfengxia Forest Park & National & Taibai County & 4360 & 92 & $4 \mathrm{~A}$ \\
\hline 5 & Honghegu Forest Park & $\begin{array}{c}\text { Province } \\
\text {-level }\end{array}$ & Mei County & 2341 & 91.7 & $4 \mathrm{~A}$ \\
\hline 6 & Zibaishan Forest Park & $\begin{array}{c}\text { Province } \\
\text {-level }\end{array}$ & Feng County & 1120 & 95 & $3 \mathrm{~A}$ \\
\hline 7 & Xueshandong Forest Park & $\begin{array}{l}\text { Province } \\
\text {-level }\end{array}$ & Gaoxin District & 1335 & 89.9 & - \\
\hline
\end{tabular}

\section{Establishment and evaluation of assessment index system}

\subsection{Evaluation method and data source}

The ecological security evaluation of all forest parks in Baoji section of the Qinling Mountains is conducted with the help of quantitative index system and visible data to reflect the ecological security grade in parks. On the basis of forest park ecological security evaluation system proposed by Wang Chaohui, this thesis adopts the PSR model to evaluate and study the ecological security of all forest parks, i.e. pressure-state-response model ${ }^{[8]}$. In this model, $P$ denotes the pressure imposed by tourists and forest park construction to the natural environment; $S$ means that the ecological and environmental state of forest parks inevitably changes due to the pressure; and $R$ denotes the response to the forest ecological change due to the pressure imposed by tourists and forest park construction.

In this research, in-depth investigation is conducted to obtain actual information of all forest parks in Baoji section of the Qinling Mountains according to the sustainable development criteria for ecological tourism in

Table 2. Ecological security index system and weight distribution of forest parks in the study area

\begin{tabular}{|c|c|c|c|c|c|c|c|c|}
\hline$\frac{0}{\frac{0}{0}} \underset{\pi}{\pi}$ & 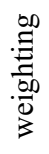 & 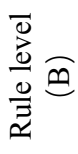 & $\begin{array}{l}\stackrel{00}{\Xi} \\
.00 \\
\frac{010}{2} \\
3\end{array}$ & 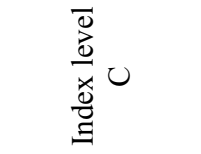 & 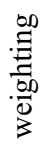 & $\begin{array}{l}\text { 흥 } \\
0 \\
\frac{1}{0} \\
0 \\
0 \\
0\end{array}$ & 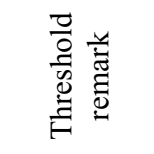 & 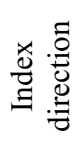 \\
\hline \multirow{2}{*}{ 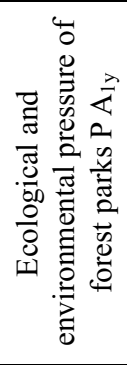 } & \multirow{2}{*}{$\begin{array}{l}\hat{b} \\
\infty \\
\tilde{0}\end{array}$} & \multirow{2}{*}{ 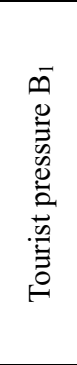 } & \multirow{2}{*}{$\begin{array}{l}n \\
n \\
\tilde{n}\end{array}$} & 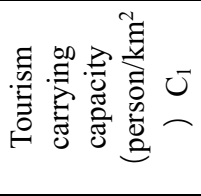 & $\stackrel{\substack{N \\
n}}{\tilde{n}}$ & ஓ & 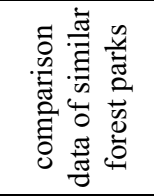 & 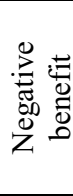 \\
\hline & & & & 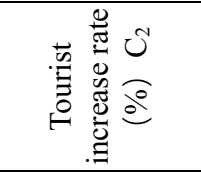 & 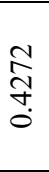 & $\begin{array}{l}\text { ํं } \\
\text { ก̊ }\end{array}$ & 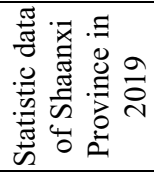 & 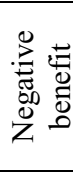 \\
\hline
\end{tabular}

Qinling; expert questionnaire method is adopted to determine the index system. The data come from Baoji Statistical Yearbook (2018), Baoji National Economic and Social Development Bulletin (2018), Baoji Environmental Monitoring Data and Environmental Status Bulletin, information provided by statistics bureau and environmental protection agency of Baoji City as well as other statistic data available on the government website, etc.

\subsection{Assessment system establishment and index weighting setup of forest parks in Baoji section of the Qinling Mountains}

The comprehensive index $(\mathrm{O})$ of ecological security grade of the forest parks in Baoji section of the Qinling Mountains is taken as the overall target level to characterize the ecological security coefficient grade. The weighting of all index system is scored according to expert comments. Feedback on index with big difference of score is given. All types of index values tend to be consistent after modification of expert comments. The distribution of index weighting is finally determined as shown in Table 2. 


\begin{tabular}{|c|c|c|c|c|c|c|c|c|}
\hline & & \multirow{2}{*}{ 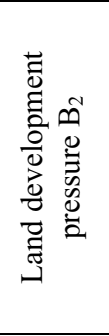 } & \multirow{2}{*}{$\underset{\substack{\hat{n} \\
0}}{0}$} & 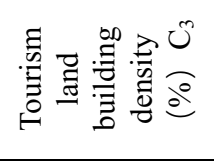 & $\frac{\substack{0 \\
0 \\
0}}{0}$ & $\begin{array}{l}\text { ठें } \\
\text { ì }\end{array}$ & 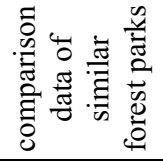 & 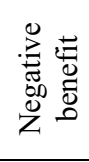 \\
\hline & & & & 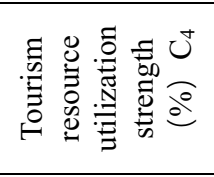 & $\begin{array}{l}\hat{0} \\
\text { o } \\
\vdots \\
0\end{array}$ & ठें & 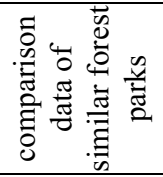 & 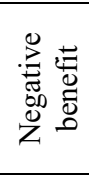 \\
\hline & & 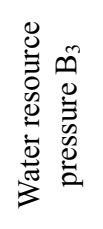 & $\begin{array}{l}\stackrel{\infty}{0} \\
\stackrel{0}{0}\end{array}$ & 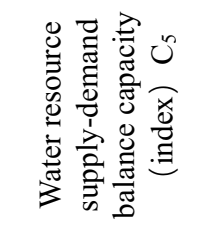 & - & - & 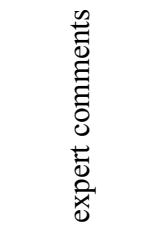 & 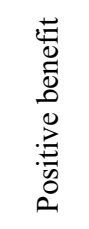 \\
\hline & & 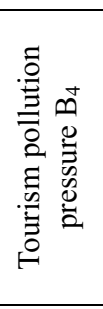 & 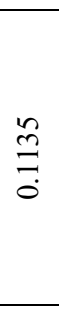 & 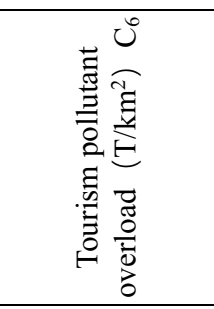 & - & ถి & 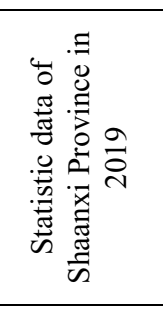 & 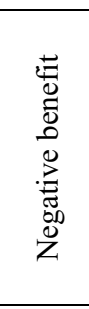 \\
\hline & & $\begin{array}{l}\stackrel{n}{0} \\
\stackrel{0}{\Xi}\end{array}$ & & 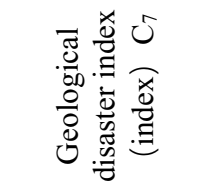 & 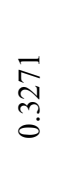 & - & 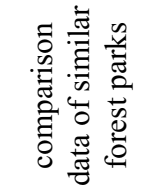 & 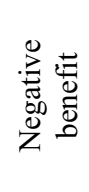 \\
\hline & & 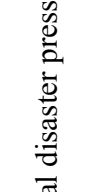 & $\begin{array}{l}n \\
\tilde{o} \\
0 \\
0\end{array}$ & 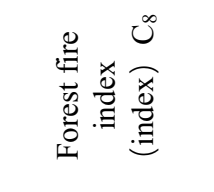 & $\begin{array}{l}\overrightarrow{\widetilde{N}} \\
\text { o }\end{array}$ & - & 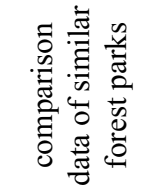 & 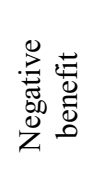 \\
\hline & & $\begin{array}{l}\text { 売 } \\
\text { Z }\end{array}$ & & 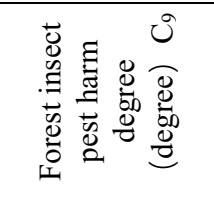 & $\begin{array}{l}\frac{\infty}{n} \\
\stackrel{n}{0}\end{array}$ & $\begin{array}{l}\frac{\vec{t}}{.00} \\
\frac{.00}{\omega}\end{array}$ & 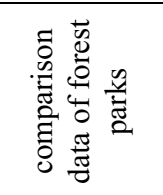 & 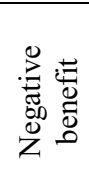 \\
\hline & & $\begin{array}{l}\infty \\
0 \\
\Xi\end{array}$ & & 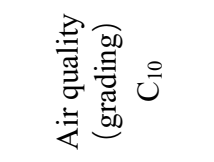 & $\frac{n}{n}$ & 节 & 岁े & 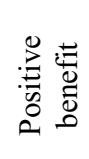 \\
\hline 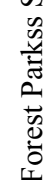 & & 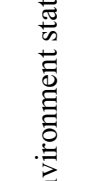 & 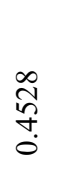 & 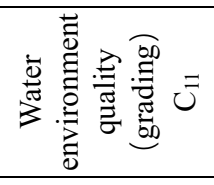 & ڤ̊n & 离 & $\begin{array}{l}\infty \\
\tilde{\infty} \\
\infty \\
\tilde{0}\end{array}$ & 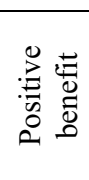 \\
\hline 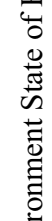 & 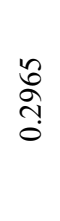 & 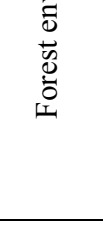 & & 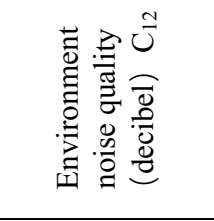 & $\begin{array}{l}\hat{\delta} \\
\text { ָे. } \\
o\end{array}$ & $\begin{array}{l}q \\
\text { ஜै } \\
\text { n } \\
v\end{array}$ & 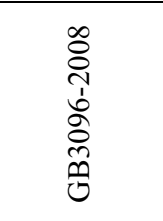 & 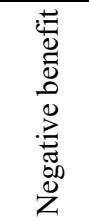 \\
\hline 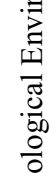 & & 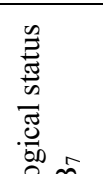 & $\underset{\mathfrak{f}}{\stackrel{\mathbb{f}}{ }}$ & 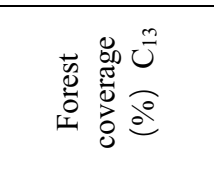 & $\begin{array}{l}\text { ڤิ } \\
\text { ָิ }\end{array}$ & ڤ̊ำ & 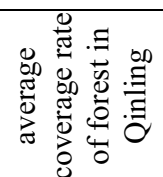 & 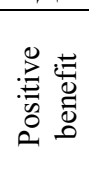 \\
\hline ư & & 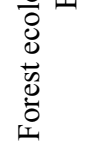 & $\ddot{0}$ & 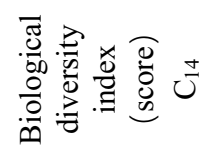 & $\begin{array}{l}\hat{c} \\
\text { n. } \\
\text { on }\end{array}$ & $\triangleright$ & 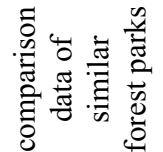 & 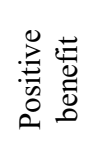 \\
\hline
\end{tabular}




\begin{tabular}{|c|c|c|c|c|c|c|c|c|}
\hline & & & & 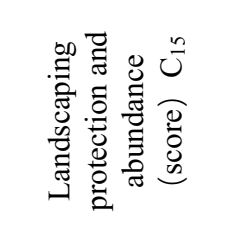 & $\begin{array}{l}\vec{f} \\
\stackrel{n}{6}\end{array}$ & $\triangleright$ & 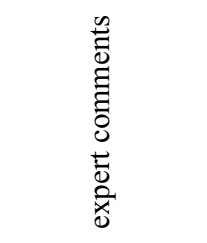 & $\begin{array}{l}\overrightarrow{0} \\
0 \\
0 \\
0 \\
0 \\
. \\
0 \\
0 \\
0\end{array}$ \\
\hline \multirow{10}{*}{ 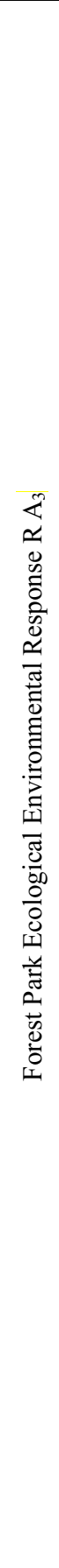 } & \multirow{10}{*}{$\frac{\mathbb{N}}{n}$} & \multirow{2}{*}{ 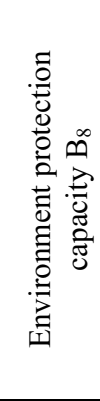 } & \multirow[t]{2}{*}{ 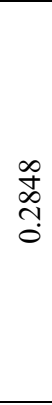 } & 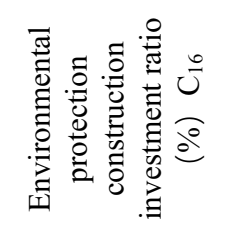 & 突 & $\stackrel{\circ}{\circ}$ & 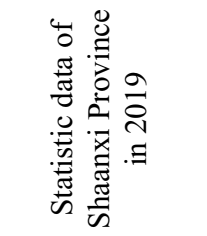 & 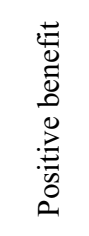 \\
\hline & & & & 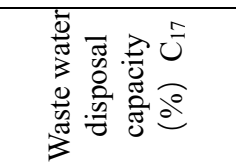 & $\begin{array}{l}\text { iे } \\
\text { के } \\
\text { वे }\end{array}$ & $\stackrel{\circ}{0}$ & 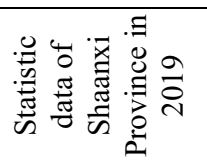 & 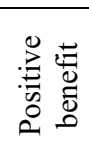 \\
\hline & & \multirow{3}{*}{ 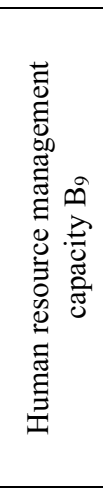 } & \multirow{3}{*}{$\frac{\pi}{\frac{n}{5}}$} & 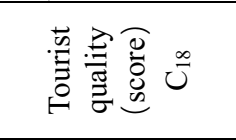 & $\underset{\infty}{\infty}$ & 8 & 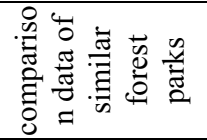 & 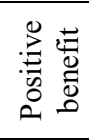 \\
\hline & & & & 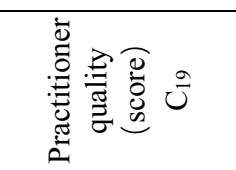 & $\begin{array}{l}0 \\
\stackrel{0}{0} \\
\cdots \\
0\end{array}$ & 8 & 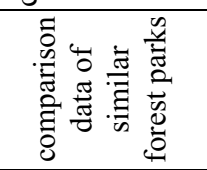 & 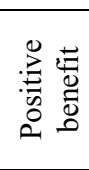 \\
\hline & & & & 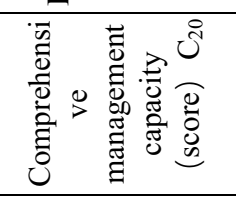 & 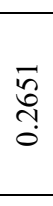 & 8 & 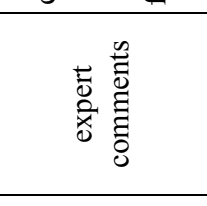 & 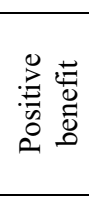 \\
\hline & & \multirow{3}{*}{ 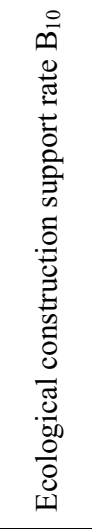 } & \multirow{3}{*}{ 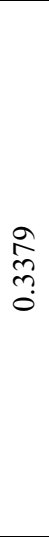 } & 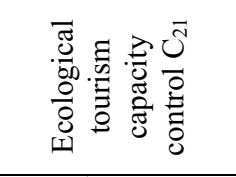 & $\begin{array}{c}\overrightarrow{7} \\
\text { ț } \\
0\end{array}$ & \& & 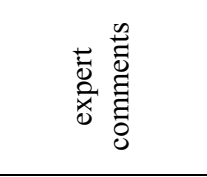 & $\begin{array}{l}\text { 总营 } \\
0.00 \\
0.00\end{array}$ \\
\hline & & & & 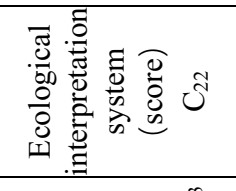 & $\frac{m}{m}$ & $\infty$ & 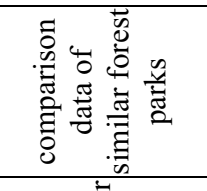 & 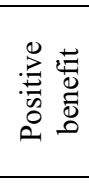 \\
\hline & & & & 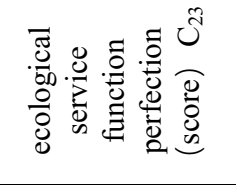 & 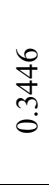 & 8 & 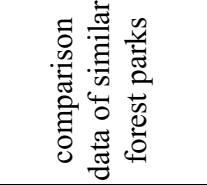 & 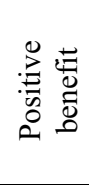 \\
\hline & & \multirow{2}{*}{ 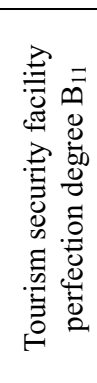 } & \multirow{2}{*}{ 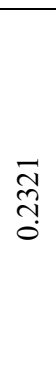 } & 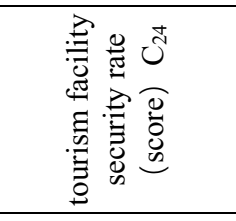 & $\begin{array}{l}\vec{\infty} \\
\infty \\
\overbrace{}^{2}\end{array}$ & $\triangleright$ & 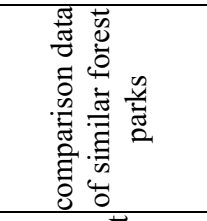 & 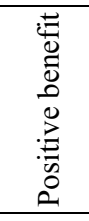 \\
\hline & & & & 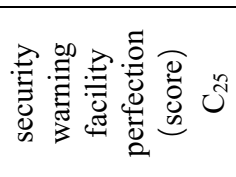 & $\stackrel{\vartheta}{\ni}$ & $\infty$ & 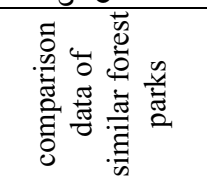 & 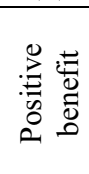 \\
\hline
\end{tabular}

\subsection{Calculation method}

There are many types of indicators in the ecological security assessment system of forest parks, and the denotation of the units of indicator values is different, so it is necessary to standardize all assessment indicators, including positive benefit indicators and negative benefit indicators, which are handled respectively according to the following process ${ }^{[8]}$.

It is assumed that $X_{i}\left(X_{i}=1,2,3, \ldots\right)$ is the index value of the $f$ indicator, $Y_{\mathrm{i}}$ is the index threshold of the $f$ indicator, and $\mathrm{S}\left(\mathrm{X}_{\mathrm{i}}\right)$ is the security index of the indicator. 
Among these indicators, the positive benefit indicator is one which is better if it is larger, and the set threshold is the lower limit of the forest park ecological security.

If $X_{i} \geq Y_{i}, \quad S\left(X_{i}\right)=1$, if $X_{i}<Y_{i}, \quad S\left(X_{i}\right)=X_{i} / Y_{i}$.

The negative benefit indicator is one which is better if it is smaller, and its set threshold is the upper limit of the forest park ecological security.

If $X_{i} \geq Y_{i}, \quad S\left(X_{i}\right)=X_{i} / Y_{i}$, if $X_{i}<Y_{i}, \quad S\left(X_{i}\right)=1$.

After handling the positive benefit and negative benefit indicators through above procedures, the ecological security index of the forest park can be calculated by the following formula:

$$
\mathrm{S}(0)=\sum_{i=1}^{24} W\left(R_{i}\right) * S\left(R_{i}\right)
$$

Where $\mathrm{R}_{\mathrm{i}}$ denotes the $i$ indicator, $\mathrm{S}\left(\mathrm{R}_{\mathrm{i}}\right)$ denotes the ecological security index of the $i$ indicator, $\mathrm{W}\left(\mathrm{R}_{\mathrm{i}}\right)$ denotes the weighting of the $i$ indicator, and $\mathrm{S}(\mathrm{O})$ denotes the security index of the forest park.

\subsection{Evaluation results}

In this research, the ecological security index of forest parks is divided into four grades according to Tang Peng's classification criteria of ecological security grade of forest parks, including security $(0.75 \leq \mathrm{S}<1)$, relative security $(0.5 \leq \mathrm{S}<0.75)$, slight insecurity $(0.25 \leq \mathrm{S}<0.5)$, and insecurity $(0 \leq \mathrm{S}<0.25)^{[6]}$.

Through calculation, the overall ecological security index of seven forest parks, ecological environment pressure (P), ecological environment state (S) and ecological environment response of all forest parks in Baoji section of the Qinling Mountains are given in Figure 2.

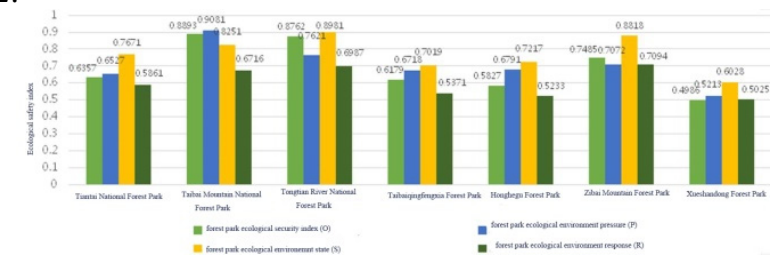

Figure 2. Distribution of ecological security index of forest parks in the study area

The above figure indicates that among the forest parks in Baoji section of the Qinling Mountains, the scores of Taibai Mountain National Forest Park (0.8893) and Tongtian River National Forest Park (0.8762) fall into the category of security, while the scores of Tiantai Mountain National Forest Park (0.6357), Taibaiqingfengxia National Park (0.6179), Honghegu Forest Park (0.5827) and Zibaishan Forest Park (0.7485) fall into the category of relative security, and the score of Xueshandong Forest Park (0.4972) belongs to slight insecurity. The performance of ecological environment state of seven forest parks is better than that of the other two indexes, indicating that forest parks are featured by high ecological grade and good ecological basis, but the tourism development and construction of forest parks have a great influence on the ecological system of forest parks. The index of ecological environment response and ecological environment pressure is low, indicating that the ecological environment pressure of forest parks is large, and the protection effect of government officials and tourists for forest ecological environment is weak.

In this research, indicators with low safety index among 25 indicators are selected to summarize problems during protection and construction of seven forest parks. Among 9 indexes of the ecological environment pressure (P) in seven forest parks, the safety indexes of tourism carrying capacity, tourism land building density and tourism resource utilization strength are low. Among 6 indicators of ecological environment state $(S)$ in the forest parks, the safety index of landscaping protection and abundance is low. Among 10 indicators of ecological environment response (R), the safety indexes of practitioner quality, tourism quality, forest park comprehensive management capacity, ecological tourism carrying capacity control, ecological interpretation system and ecological service perfection is low.

\section{Summary on risks of ecological safety in forest parks}

Four problems are summarized through evaluation on 25 indicators of ecological safety in seven forest parks according to current conditions of construction and development of forest parks. First, forest parks are simply intended to attract a larger population of tourists. The tourism environment capacity of forest parks is evaluated, proving that park managers cannot make adjustment according to seasons. In holidays and peak seasons, the number of tourists may far exceed the ecological carrying capacity of forest parks, thus degrading the biological diversity, and the degradation rate of landscaping appreciation and tourism facility is high. Secondly, due to visual conflicts of artificial landscaping in forest parks, the building style of tourism service facilities such as tourist service center, hotels and agritainment is inconsistent, and the building scale and density is large. Thirdly, the overall quality of managers in forest parks is low, and the overall management ability is weak, e.g. environmental governance, forest park holistic planning, tourism regulation implementation, tourist behavior control, tourist guide ability, etc. Finally, the concept of forest park ecological tourism and mass tourism is confusing, the tourist quality is low, and ecological interpretation guidance system and park ecological service facilities do not play their roles.

\section{Research on ecological safety protection of forest parks in Baoji section of the Qinling Mountains}

\subsection{Scientifically control tourism carrying capacity, and establish tourism emergency warning mechanism}

The tourism carrying capacity warning mechanism should be established in forest parks during tourism peak period and holidays to strictly control the number of tourists in 
each period. A scientific planning of tourism route should also be made in forest parks to strictly control the number of tourists in the forest park route, guide and adjust tourists in all landscape spots in the forest parks, and avoid crowding. The designated persons shall take charge of vulnerable ecological tourism resource zone. Forest parks shall be coordinated with all landscape spots in Baoji City. The electric display shall be installed in main tourism roads to guide tourists ${ }^{[10-11]}$.

The risk evaluation system and monitoring system on ecological environment of forest tourism shall be established and improved according to tourism emergency events. Full risk evaluation shall be conducted regularly for mountains and waters, rare animals and plants, tourism buildings and diets on the basis of landscaping spots. Some advanced technological tools such as satellite remote sensing, artificial intelligence and big data shall be adopted, and risk database and risk analysis judgment shall be built so as to provide basis for decision-making on emergency environment events ${ }^{[12-13]}$.

\subsection{Optimize forest space design, and control tourism building density}

The functional zones of forest parks shall be classified into natural conservation zone, buffer zone and tourist service zone, and the architectural, facility and landscaping design specifications shall be formulated for these three zones. The landscaping design is aimed to coordinate with forest environment, reflect natural distinctive character, so the forest aesthetics and ecological design ideas are integrated into forest park landscaping design. The conservation zone of rare animals and plants shall be strictly controlled, and the red line of forest park ecological protection shall be strictly followed. It is not allowed to carry out large-scale construction in park-owned and buffer area. The supporting facility building density and scale in the conservation zone shall be strictly controlled. The wastewater, waste disposal system and other supporting facilities shall be improved. Environment-protection construction materials shall be promoted. The tourist footpath and toilets shall comply with ecological requirements. The forest parks and outside cities as well as villages shall be linked through green path and landscaping bridges. The forest ecological system shall be harmonious. All types of ecological sports place and entertainment development space shall be properly built without affecting normal operation of the forest ecological system according to the tourism function of all forest parks, but the building density of tourism supporting facilities shall be strictly controlled ${ }^{[14-16]}$.

\subsection{Promote comprehensive management quality, and establish green forest management system}

The green forest management system mainly includes manager and operator quality education, forest disaster warning mechanism, forest ecological monitoring system and park management decision-making system. ${ }^{[17]}$. At first, park managers and operators must regularly organize learning and training on knowledge of forests, get familiar with forest tourism regulations, and perform management of environment monitoring. The municipal forest park management office and other departments shall regularly organize quality evaluation and inspection, play the role of guidance and supervision, and strengthen cultivation of the staff. The environment education shall be carried out for stall owners inside forest parks. The environment protection ideas shall be cultivated. Employees shall be encouraged to propose advice on environment protection. The measures on environment protection management in forest parks shall be actively implemented. Secondly, parks shall establish forest disaster warning mechanism to strictly control natural disasters such as forest fire, insect pest and geological disasters, etc. The tourism projects in parks shall be continuously monitored and systematically evaluated so as to ensure that projects may not have adverse influence on forests. Thirdly, the Internet-based management of tourism ecological and environment monitoring shall be pushed ahead, the monitoring force shall be optimized and allocated, and the tourism ecological and environment monitoring services shall be covered in forest parks. Finally, the legal forest administration management system shall be standardized, the mass engagement and expert verification mechanism of major decision shall be improved, and the goal responsibility system which is centered in ecological environment quality shall be improved ${ }^{[18]}$.

\subsection{Promote tourists' social public moralities and advocate forest green tourism}

Explicit warning and management method is necessary, or it may cause conflicts with tourists, leading to an opposite result to what it is expected. Therefore, indirect management shall be adopted together with direct management. During civilized management of tourists, green forest tourism shall be advocated and forest ecological values shall be displayed so that tourists may actively participate in civilized activities in forests. Through promotion of forest ecological protection knowledge, tourists are guided to learn relevant ecological knowledge and precautions on forests, tourists' forest ecological awareness may be constantly promoted. The green consumption style shall be greatly fostered. The forest green tourism consumption guidance shall be issued. Tourists shall be guided to foster the green consumption ideas of loving the nature, pursuing health, emphasizing environmental protection and saving resources, so as to actively transform green consumption ideas into green consumption behavior. Green tourism is advocated, ecological parking lots are built. Green and ecological travelling mode such as travelling by bicycle and by foot is advocated. The forest low-speed green traffic system is built. The level of green travelling public traffic service is promoted. In forest ecological activities, tourists' ecological environment awareness shall be strengthened. Green production and green consumption shall be guided. The good ecological moral awareness shall be fostered. People's awareness of loving and cherishing forests shall be strengthened ${ }^{[19-20]}$. The influence of Qinling forest 
culture shall be enlarged with the help of modern media. The centralized publicity on Qinling forest ecological culture protection, forest ecological experience, teaching, joint construction by communities, green camp and international cooperation shall be carried out so that attention on and protection of forests become people's spontaneous activity. The youth volunteer activity on protecting forests shall be regularly carried out. The forest protection publicity activity shall be widely carried out on the basis of World Forest Day, Bird-Loving Week, World Environment Day and low carbon day.

\section{Conclusion and expectations}

This thesis selects indexes with low safety index to analyze the ecological safety risks in forest parks in Baoji section of the Qinling Mountains on the basis of ecological safety evaluation of forest parks, and also concludes that the concept of forest park ecological tourism and mass tourism are confusing. This is not beneficial to the sustainable development of forest parks. Therefore, this thesis studies the protection path of forest ecological safety from the perspective of tourism carrying capacity control, landscaping protection and design, forest park green management, tourist tourism mode guidance, etc. It is expected that this study may draw the attention of relevant governments, forest park administrators and tourists to forest park ecology, improve existing hidden risks of ecological safety, promote ecological and environmental protection of forest parks, link forest ecological tourism, engage all people in it so that green tourism is reflected in all work of protection and development of forest parks.

\section{Acknowledgement}

Program: A general program funded by the Scientific and Technological Programs of Shaanxi Province (Program No.: 2018JQ5141)

\section{Authors}

Author: Zuo Ying, male, doctoral candidate, College of Urban and Environmental Sciences, Northwest University, $\mathrm{Xi}$ 'an; research interest: urban and regional development.

Corresponding author: Cui Yan, male, born in Xi'an, professor of the School of Architecture and Civil Engineering, Xi'an University of Science and Technology; research interest: urban and rural planning, tourism management. Email: 87086559@qq.com

\section{References}

1. Du Qingyi. Develop Qinling Forest Tourism, Promote Ecological Civilization Construction[J]. China Forestry Industry, 2016(7):181-182.

2. Wang Suicong. On Construction of Core Scenic Region of Grand Qinling Ecotourism[J], Shaanxi Forest Science and Technology, 2015(1):127-130.
3. Zhang Yanan. Forest Ecological Culture and Characteristic Research in Heilongjiang Province[J]. Forestry Science \& Technology, 2017(6):57-59.

4. Guo Zhenggui. Push ahead High-quality Development of Forest Tourism[J].Forestry and Ecology,2019(1):26-28.

5. Qu Zhongzheng. Cognition of Forest Tourism Culture Based on Beautiful China[J].Forestry and Ecology,2019(2):36-37.

6. Tang Peng. Ecological Security Evaluation of Zijin Mountain National Forest Park Based on PSR Model[J]. Jiangsu Agricultural Sciences, 2016(8):477-480.

7. Mi Feng. Evaluation of Ecological Security for the Jiufeng National Forest Park in Beijing[J]. Scientia Silvae Sinicae,2010(11):52-58.

8. Wang Chaohui. Research on Management Innovation for Ecological Security in Chinese Forest Park[J]. Resource Development \& Market,2013(2):152-155.

9. He Wenjuan. A Preliminary Assessment of Ecological Security in Maofengshan Forest Park, Guangzhou[J]. Guangdong Forestry Science and Technology,2011(3):41-45

10. Han Huiping, Chen Yimin. Discussion on Ecotourism Development of Nanjing Laoshan National Forest Park Based on SWOT Analysis[J]. China Forestry Economy,2020(2):97-100.

11. Chen Xiaojie. Study on the Development Status and Countermeasures of Forest Park Ecotourism[J]. Huahui,2019(12):175-176.

12. Xu Chengxiu. Analysis on Sustainable Development Strategy of Forest Park Ecotourism in Chenzhou City[J]. South China Agriculture,2019(18):81-82.

13. Li HongBin. Current Situation and Sustainable Development Countermeasures of Ecotourism Resources in Forest Parks[J]. Modern Horticulture,2019(8):165-166.

14. Cui Jie. Study on Ecological Tourism Landscape Planning of Forest Park[J]. Forestry Science and Technology Information,2019(2):122-124.

15. Xiao Lingling, Hu Weishan. Study on Ecotourism Master Plan of Mountain Forest Park -- a Case Study of Qishihe Forest Park in Sihui City[J]. Journal of Anhui Agricultural Sciences,2020(7):133-139.

16. Lou Yiwei, Guo Liang. Characteristics and Evaluation on Landscape Resources in Dachen Islands, Journal of Zhejiang Forestry Science and Technology,2016(3):88-93.

17. Huang Shengyan. On the Effective Strategies of Strengthening the Management of Forest Resources $[\mathrm{J}]$. Seed Scicence \& Technology,2019(9):137-137.

18. Li Zhuoyu. Study on the Construction of Urban Forest Park in the Context of Ecological Civilization[J].Xiangcunkeji, 2019(18):68-69.

19. Ding Wutong, Li Dengming. Optimization of Marketing Strategy of Langshan National Forest 
Park[J]. China Forestry Economy,2020(1):105-107.

20. Wang Huaifang. Countermeasures and Suggestions on Developing Forest Tourism in Dongpo Forest Park of Yong'an[J]. Anhui Agricultural Science Bulletin,2020(6):65-67. 